\title{
MUTATIONS OF CODON 918 IN THE RET PROTO-ONCOGENE CORRELATE TO POOR PROGNOSIS IN SPORADIC MEDULLARY THYROID CARCINOMAS
}

\author{
J. ZEDENIUS, C. LARSSON, U. BERGHOLM, J. BOVÉE, A. SVENSSON, B. HALLENGREN, \\ L. GRIMELIUS, M. BÄCKDAHL, G. WEBER, AND G. WALLIN
}

Departments of Surgery (J.Z., A.S., M.B., G.Wa.) and Molecular Medicine (J.Z., C.L., J.B., G.We.), Karolinska Hospital, Stockholm; Departments of Cancer Epidemiology (U.B.) and Pathology (L.G.), Uppsala University Hospital, Uppsala; Department of Endocrinology (B.H.), Malmö University Hospital, Malmö, Sweden.

\begin{abstract}
The hereditary multiple endocrine neoplasia syndromes types $2 A$ and B (MEN 2A and B) were recently linked to germline mutations in the RET proto-oncogene, altering one of five cysteine residues in exon 10 or 11 (MEN 2A), or substituting a methionine for a threonine at codon 918 in exon 16 (MEN 2B). The latter mutation also occurs somatically in some sporadic medullary thyroid carcinomas (MTC), and has in a previous study been correlated with a less favorable clinical outcome. In the present study, $46 \mathrm{MTCs}$ were selected for investigation of the codon 918 mutation. The mutation was found in 29 tumors (63\%), and was significantly correlated with a poor outcome, with regard to distant metastasis or tumor recurrence $\left(\mathrm{p}<10^{-4}\right)$. Two tumors showed multifocal growth and $\mathrm{C}$-cell hyperplasia, and these patients were therefore also investigated for germline mutations in exons 10,11 and 16. The codon 918 mutation was found only in the tumors, thus of somatic origin. The RET codon 918 mutation may have prognostic impact, and therefore preoperative assessment may influence decision-making in the treatment of patients suffering from MTC.
\end{abstract}

Multiple endocrine neoplasia type 2A (MEN 2A) is an autosomal dominant inherited neoplasia syndrome with a high degree of penetrance and variable expressivity. It is characterized by the association of medullary thyroid carcinoma (MTC) with pheochromocytoma $(\approx 50 \%)$ and in some cases hyperparathyroidism $(\approx 20 \%)$. The familial forms constitute about $25 \%$ of all cases of MTC. MTC is seen in virtually all MEN $2 \mathrm{~A}$ patients and usually differs from the sporadic counterparts in that the tumors are multifocal and are preceded by general C-cell hyperplasia (1). MEN 2B is characterized by the association of MTC and pheochromocytoma with a distinct phenotype: mucosal neuromas, intestinal ganglioneuromatosis and neurological disturbances (2). About half of the cases of MEN 2B appear to be new mutations, i.e. they have no prior family history of the disease but their children have a $50 \%$ risk of developing the disease. Often, the MTC in these patients is very aggressive and occurs at early age (3).

Recently, it was shown that the RET proto-oncogene (RET), coding for a receptor tyrosine kinase expressed in neural crestderived tissue, is the gene responsible for MEN 2A and MEN 2B when germline mutations were identified in affected patients but not in their unaffected relatives. Nearly all mutations related to MEN 2A and isolated familial MTC involve the alteration of one of five cysteine residues within exons 10 and $11(4,5)$. MEN 2B patients instead show a mutation substituting a methionine with a threonine at codon 918 in exon 16 of RET (6-8). It has recently been shown that these mutations activate RET to function as a dominant transforming gene (9).

As in several other disorders, the germline mutations responsible for the MEN2 syndromes are also found in the sporadic counterparts. In the case of sporadic MTC, somatic mutations have almost exclusively affected codon 918 in exon 16 of RET, a mutation similar to that found in constitutional DNA of MEN
2B patients. About one third of sporadic MTCs have this mutation $(6,10)$. In an initial study of sporadic MTCs, the occurrence of this mutation was related to a less favorable clinical outcome (11). As the number of MTCs was small, in the present study we have extended the sample to 46 patients with clinically sporadic MTC, and have correlated the presence of RET codon 918 mutation with the patients clinical outcome.

\section{Material and methods}

\section{Patients}

Forty-six patients with clinically sporadic MTC diagnosed 1960-1994 were selected for this study. The MTC was judged as sporadic if there was no family history of disease related to any of the MEN 2 syndromes, and if the patient did not exhibit any clinical signs of an MEN $2 A$ or B phenotype. All tumors were re-evaluated regarding multifocal growth pattern and C-cell hyperplasia: 44 tumors did not show any of these signs, while two did and therefore were selected for further mutational analysis (see below).

The patients were divided into two groups:

Group I $(n=15)$. No sign of residual or recurrent disease following initial surgical treatment. Patients were regarded as disease-free if clinical and radiological examination did not show any signs of tumor, and if basal serum calcitonin levels were normal. Calcitonin assays have varied during time. Presently, a radioimmunoassay is being used (normal value $<28 \mathrm{pmol} / \mathrm{l}$ ). Mean age at diagnosis was 53 years (25-76 yrs). Median follow-up time was 123 months (72-371 mos.).

Group II $(n=31)$. Incurable disease at initial operation $(n=6)$, or recurrent disease with either local recurrence or distant metastases $(n=24)$, or elevated post-operative basal calcitonin levels $(n=1)$. Mean age at diagnosis was 55 years (12-79 yrs). In the recurrence group, median disease-free interval was 11 months $(1-143 \mathrm{mos}$.). The patient with elevated basal calcitonin level $(\approx 350 \mathrm{pmol} / \mathrm{l})$ was 61 years at diagnosis, and has been followed for 26 months without exhibiting any 
other clinical symptoms of recurrence. This patient's tumor was one of the two exhibiting multifocal growth and C-cell hyperplasia. The other patient with a tumor showing these phenomena was a 23 year old male with extensive metastases at initial operation.

\section{DNA extraction}

Three patients from group I and six patients from group II have been reported previously (11). Tumor DNA was, in these cases, extracted from fresh frozen tissue. In all other cases, tumor DNA was extracted from archival paraffin embedded tissue. In short, four $5 \mu \mathrm{m}$ sections were de-paraffinized with lemonene, and tumor cell areas dissected into tubes containing lysis buffer $(0.05 \mathrm{M}$ Tris- $\mathrm{HCl} \mathrm{pH} 7.9,0.15 \mathrm{M} \mathrm{NaCl}$ $5 \mathrm{mM}$ EDTA, $1 \%$ SDS, Proteinase $\mathrm{K} 500 \mu \mathrm{g} / \mathrm{ml}$ ). The samples were then incubated at $+45^{\circ} \mathrm{C}$ overnight, followed by phenol-chloroform extraction and ethanol precipitation.

\section{Polymerase chain reactions (PCR) and mutation analysis}

Amplification of exon 16 of RET was done by PCR using the primers 16F (5'-AGG GAT AGG GCC TGG GCT TC-3') and 16R (5'-TAA CCT CCA CCC CAA GAG AG-3') as previously described (11). If the DNA used in this PCR was obtained from archival tumor tissue, another PCR was performed using nested primers, one of them biotinylated: N16F (5'-CTT CAA TGC TTT ATT CCA TCT TCT C-3') and N16R (5'-CAA CAC CCA CAC TTA CAC ATC AC-3'), resulting in a 117 basepair product. Nested PCR was done with $8 \mu \mathrm{l}$ of first PCR product as template and $1.5 \mathrm{mM} \mathrm{MgCl}_{2}$ in a final volume of $100 \mu \mathrm{L}$. Thermal cycling conditions were incubation at $95^{\circ} \mathrm{C}$ for $4 \mathrm{~min}, 35$ step cycles at $95^{\circ} \mathrm{C}$ for $30 \mathrm{~s}$, at $54^{\circ} \mathrm{C}$ for $30 \mathrm{~s}$, and at $72^{\circ} \mathrm{C}$ for $30 \mathrm{~s}$, with a final extension for $5 \mathrm{~min}$ at $72^{\circ} \mathrm{C}$. Purified PCR products (Wizard PCR Prep, Promega) were subjected to $(A)$ sequencing from both DNA strands using Dynabeads (Dynal) and the Sequenase kit (USB), and (B) restriction enzyme digest by $F o k 1$ as described (11). Sequence reactions were run on $6 \%$ denaturing polyacrylamide gels, and Fok1 digests on $1 \%$ LMP $/ 1 \%$ normal agarose gels.

Blood, normal thyroid tissue and tumor tissue from two patients were subjected to sequence analysis of RET exons 10,11 and 16 for reasons described above. These analyses were performed as described (11).

\section{Statistical analysis}

Fisher's exact test was used for statistical analysis.

\section{Results}

In this study, genomic DNA from 46 sporadic MTCs was analyzed by direct sequencing of RET exon 16 . To increase the reliability of the analysis both DNA strands were sequenced.
Informative results were obtained from 41 of the tumors. In the remaining five cases, mutation analysis was based on Fok 1 digest results.

Of the 46 patients with MTCs selected for the study, 31 $(67 \%)$ showed signs of incurable or recurrent disease. Of these, $26(84 \%)$ showed the mutation in codon 918 of RET, which substitutes a methionine (ATG) with a threonine (ACG). Of the tumors from the 15 patients without recurrent MTC, 12 (80\%) did not have any codon 918 mutation. A statisitically significant correlation was made between the presence of the RET codon 918 mutation and a poor outcome $\left(p<10^{-4}\right)$ (Table 1). The mean age of the patients with mutations in the tumor tissue was similar to that in patient group II. The mutations were apparently heterozygous, as the wildtype sequence in all cases was visible to at least the same extent as the mutant (Figure 1).

The three patients in Group I whose tumors showed the RET codon 918 mutation, did not significantly differ from the patients in Group II with regard to age at diagnosis, tumor size, or the surgical procedures used. Similarly, the five patients in Group II whose tumors did not show the mutation, were not different from the patients in Group I with regard to clinicopathological data, or surgical treatment.

In the two cases exhibiting multifocal tumor growth and $\mathrm{C}$ cell hyperplasia, no mutation was found in exons 10,11 and 16 when analyzing DNA from blood and normal thyroid tissue. However, the codon 918 mutation was in both cases found in the tumor tissue, thus proven to be of somatic origin. These patients were 23 and 61 years of age at diagnosis, respectively.

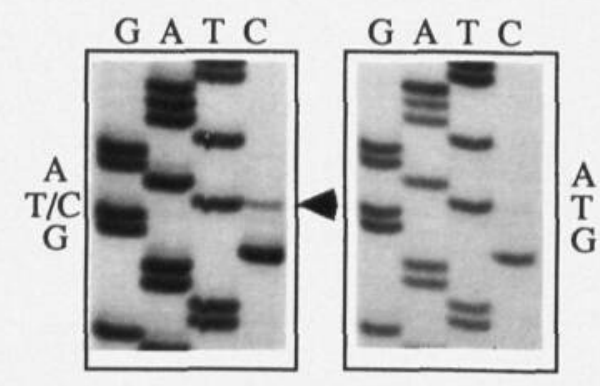

FIG. 1. Autoradiograms of the partial sequencing of RET exon 16 in two tumors. In the tumor to the left, a methionine (ATG) is substituted with a threonine (ACG) at codon 918, as indicated by the arrow.

TABLE 1. Summary of the RET codon 918 mutations in the 46 sporadic MTCs with regard to the patients clinical outcome. The correlation is statistically significant $\left(p<10^{-4}\right)$. For definition of patient groups, see Material and Methods.

\begin{tabular}{lccc}
\hline & Group I. Disease-free & Group II. Recurrent disease & Total \\
\hline No mutation & 12 & 5 & 17 \\
Mutation & 3 & 26 & 29 \\
Total & 15 & 31 & 46 \\
\hline
\end{tabular}




\section{Discussion}

Somatic RET mutations have previously been reported in sporadic MTCs $(6,10,11)$. These mutations affect codon 918, substituting a methionine (ATG) with a threonine (ACG), the same mutation found constitutionally in virtually all MEN 2B patients. About one third of sporadic MTCs so far reported exhibit this mutation. Recently, a missense mutation of codon 768 was reported in some sporadic MTCs without the codon 918 mutation (12). With further studies, it may turn out that the majority of MTCs carry a mutation somewhere in RET.

In this study, we have only investigated for the codon 918 mutation. The RET codon 918 mutation was strongly correlated to poor prognosis $\left(\mathrm{p}<10^{-4}\right)$. The comparably high proportion of tumors with this mutation in the material $(63 \%)$ probably reflects our selection of patients with mainly recurrent MTC, and is thus not in disagreement with earlier reports where a lower general mutation rate was given $(6,10)$.

The exact mechanism by which this mutation acts on the cellular level is not clearly understood. However, recently presented data show that this specific mutation, which alters an amino acid within a highly conserved substrate-recognition site in the catalytic core of the tyrosine kinase, probably results in a shift of substrate specificity (9). A methionine at this site is found in cytoplasmatic tyrosine kinases rather than in receptor tyrosine kinases, e.g. RET. It has been shown that the MEN 2B mutation in vitro results in a shift in peptide substrate specificity, thus involving downstream signalling proteins not normally activated by RET (13). This phenomenon could explain the reason for MEN 2B tumors being more aggressive than their counterparts $(2,3)$. Subsequently, it may explain why the mutation is correlated to poor prognosis in sporadic MTC.

Our study shows that the detection of a RET codon 918 mutation in sporadic MTC indicates a high risk of recurrence. Pre-operative detection of the mutation may implicate the need for extended lymph node dissection at initial surgery, or a more careful search for yet undetected metastases. (A rapid method for pre-operative analysis of RET codon 918 mutation on fine needle aspirates will be presented elsewhere, and is available from the authors.) RET codon 918 mutation analysis together with other clinical data will help individualize the treatment for patients with MTC. In addition, the detection of this somatic mutation makes it highly unlikely that the patient carries a germline mutation elsewhere in RET which predisposes to pheochromocytoma (10). The risk of an undetected pheochromocytoma by the time of surgery would therefore be markedly reduced. As an example, the only two tumors in this material resembling a hereditary MTC variant (multifocal growth pattern and C-cell hyperplasia) were not due to a germline mutation in RET, but showed the somatic codon 918 mutation. However, since a stem cell mutation due to an early mitotic event cannot be excluded, it may be appropriate to screen for the corresponding germline mutation in the offspring of such patients.

\section{Acknowledgments}

We wish to thank Dr Lennart Bondesson for contributing some of the tumor specimens. This study was supported by the Swedish Medical
Research Council $(0102,2330)$, the Stockholm Cancer Society (93:115), the Swedish Cancer Foundation, the Swedish Society of Medicine, the Lars Hierta, Magnus Bergwall and the Torsten and Ragnar Söderberg Foundations.

\section{References}

1. Block MA, Jackson CE, Greenawald KA, Yott JB, Tashjian AH. 1980 Clinical characteristics distinguishing hereditary from sporadic medullary thyroid carcinoma. Arch Surg. 115:142-148.

2. Khairi MRA, Dexter RN, Burzynski NJ, Johnston C.C. 1975 Mucosal neuroma, pheochromocytoma, and medullary thyroid carcinoma: multiple endocrine neoplasia type 3. Medicine. 54:85112.

3. Norton JA, Froome LC, Farrell RE, Wells SA Jr. 1979 Multiple endocrine neoplasia type $\mathrm{Il} b$ - the most aggressive form of medullary thyroid carcinoma. Surg Clin North Am. 59:109-118.

4. Mulligan LM, Kwok JBJ, Healey CS, et al. 1993 Germ-line mutations of the RET proto-oncogene in multiple endocrine neoplasia type 2A. Nature. 363:458-460.

5. Donis-Keller H, Dou S, Chi D, et al. 1993 Mutations in the RET proto-oncogene are associated with MEN $2 \mathrm{~A}$ and FMTC. Hum Mol Genet. 2:851-856.

6. Hofstra RMW, Landsvater RM, Ceccherini I, et al. 1994 A mutation in the RET proto-oncogene associated with multiple endocrine neoplasia type $2 \mathrm{~B}$ and sporadic medullary thyroid carcinoma. Nature. 367:375-376.

7. Carlson KM, Dou S, Chi D, et al. 1994 Single missense mutation in the tyrosine kinase catalytic domain of the RET protooncogene is associated with multiple endocrine neoplasia type 2B. Proc Natl Acad Sci USA. 91:1579-1583.

8. Eng C, Smith DP, Mulligan LM, et al. 1994 Point mutation within the tyrosine kinase domain of the RET proto-oncogene in multiple endocrine neoplasia type $2 \mathrm{~B}$ and related sporadic tumours. Hum Mol Genet. 3:237-241.

9. Santoro M, Carlomagno F, Romano A, et al. 1995 Activation of RET as a dominant transforming gene by germline mutations of MEN 2A and MEN 2B. Science. 267:381-383.

10. Eng C, Mulligan LM, Smith DP, et al. 1995 Mutation of the RET protooncogene in sporadic medullary thyroid carcinoma. Genes Chromosom Cancer. 12:209-212.

11. Zedenius J, Wallin G, Hamberger B, Nordenskjöld M, Weber G, Larsson C. 1994 Somatic and MEN 2A de novo mutations identified in the RET proto-oncogene by screening of sporadic MTC:s. Hum Mol Gen. 3:1259-1262.

12. Eng C, Smith DP, Mulligan LM, et al. 1995 A novel point mutation in the tyrosine kinase domain of the RET proto-oncogene in sporadic medullary htyroid carcinoma and in a family with FMTC. Oncogene. 10:509-513.

13. Songyang Z, Carraway III KL, Eck MJ, et al. 1995 Catalytic specificity of protein-tyrosine kinases is critical for selective signalling. Nature. 373:536-539. 